\title{
Two Types of Distributed CFAR Detection Based on Weighting Functions in Fusion Center for Weibull Clutter
}

\author{
Amir Zaimbashi \\ Department of Electrical and Electronics Engineering, Shahid Bahonar University, Kerman, Iran \\ Correspondence should be addressed to Amir Zaimbashi; a.zaimbashi@gmail.com
}

Received 22 August 2012; Accepted 8 October 2012

Academic Editor: Jie Zhou

Copyright (C) 2013 Amir Zaimbashi. This is an open access article distributed under the Creative Commons Attribution License, which permits unrestricted use, distribution, and reproduction in any medium, provided the original work is properly cited.

\begin{abstract}
Two types of distributed constant false alarm rate (CFAR) detection using binary and fuzzy weighting functions in fusion center are developed. In the two types of distributed detectors, it was assumed that the clutter parameters at the local sensors are unknown and each local detector performs CFAR processing based on ML and OS CFAR processors before transmitting data to the fusion center. At the fusion center, received data is weighted either by a binary or a fuzzy weighting functions and combined according to deterministic rules, constructing global test statistics. Moreover, for the Weibull clutter, the expression of the weighting functions, based on ML and OS CFAR processors in local detectors, is obtained. In the binary type, we analyzed various distributed detection schemes based on maximum, minimum, and summation rules in fusion center. In the fuzzy type, we consider the various distributed detectors based on algebraic product, algebraic sum, probabilistic OR, and Lukasiewicz t-conorm fuzzy rules in fusion center. The performance of the two types of distributed detectors is analyzed and compared in the homogenous and nonhomogenous situations, multiple targets, or clutter edge. The simulation results indicate the superiority and robust performance of fuzzy type in homogenous and non homogenous situations.
\end{abstract}

\section{Introduction}

The essential problem in radar detection is to determine whether or not a target is present in a special region based on single sensor detection schemes or distributed sensor detection schemes. Distributed detection schemes are needed when system performance factor such as speed, reliability against failure of individual receivers, survivability, increase in the number of targets under consideration, and constraint over the communication bandwidth are taken into account. When the background clutter is nonstationary, detection with the fixed threshold results in an intolerable increase of the false alarm probability or in a decrease of the probability of detection. Therefore, adaptive threshold techniques are required in order to maintain a nearly constant false alarm rate (CFAR). A variety of CFAR techniques are developed according to clutter models and logic used to estimate the unknown clutter parameters. Some examples are CA CFAR [1], ML CFAR [2], OS CFAR [3, 4], OSGO, and OSSO [5]. Barkat and Varshney [6] extended the CFAR technique to the distributed detection. They analyzed the CA CFAR detection with multiple sensors and fusion center. Namely, each CA CFAR detector transmits a binary decision to the fusion center where a final decision is made based on AND or OR rule. Distributed OS CFAR detectors with the AND or the OR fusion rules is considered by Üner and Varshney [7]. Amirmehrabi and Viswanathan [8] proposed a scheme called S+OS based on the local test statistics, which instead of the binary decision; each sensor transmits the sample from the test cell and a designated order statistics from the available set of the reference observations surrounding the test cell to the fusion center. In [9], a new distributed CFAR test called the normalized test statistics (NTS) was proposed. In this test, the sum of local test statistics is compared with a threshold to decide the presence/absence of a target. It was shown in [9] that NTS provides a considerable performance gain over OR or AND fusion rules under the condition of large signal-toclutter power ratio and moderate shape parameter values of the Weibull clutter. Reference [9] also proposed three types of distributed CFAR detection based on local test statistics; 
they are called R (ratio), S (substrate), and P (pulse) schemes. All of proposed detectors in [9] have used the sample in test cell and an estimation of clutter power to form local test statistics. Distributed detection of signals in non-Gaussian clutter is also considered in $[10,11]$. However, it is necessary to find more effective local processing measures and effective fusion rules in fusion center to improve the distributed detection. In [12], Hammoudi and Soltani considered the problem of distributed CA CFAR and OS CFAR detections using fuzzy spaces and fuzzy fusion rules in Gaussian clutter. Namely, each local sensor computes the value of membership functions to the false alarm space from the sample of the reference cells and transmits it to the fusion center where the final decision is made based on fuzzy fusion rules. It was shown in [12] that algebraic product fuzzy fusion rules provide a better performance than those of the OR or AND under the condition of the Gaussian clutter.

In this paper, two general types of distributed detectors based on binary and fuzzy weighting functions in fusion center are developed. In the binary type, the received data in the fusion center weighted according to a binary weighting functions, whereas in the fuzzy type it is fuzzy weighting functions. In the two types of distributed detectors, it was assumed that the clutter parameters at the local sensors are unknown and each local detector performs CFAR processing before transmitting data to the fusion center. In the binary type, we analyzed various distributed detection scheme based on ML (OS) CFAR processor in local detectors and maximum, minimum, and summation rules in fusion center. The employment of the binary weighting functions, in the binary type, certainly causes significant loss of information in the fusion center. In order to reduce this imperfection, in the fuzzy type the fuzzy weighting functions is used. In this type, we consider the various distributed detectors based on ML (OS) CFAR processor in local detectors and algebraic product, algebraic sum, probabilistic OR, and Lukasiewicz tconorm fuzzy rules in fusion center.

The paper is organized as follows. In Section 2, for a two-sensor network, first we introduce a general structure for distributed CFAR detection, and then two types of distributed CFAR detection based on binary and fuzzy weighting functions in fusion center are developed. Section 3 contains performance comparisons of various schemes based on the simulation study in homogenous and nonhomogenous situations. A summary and the conclusions derived from this study are presented in Section 4.

\section{Detection Problem Statement}

Consider a two-sensor distributed network. Here $x_{i j}$ is the observation (excluding the test sample) and $x_{i 0}$ is the sample in the test cell, where $i=1,2$ indicates the number of the sensors, and $j=1,2, \ldots, N_{i}$ represents the sample number in the range cell available to the $i$ th sensor. It is assumed that both the sensors scan the same search environment and $x_{i 1}, x_{i 2}, \ldots, x_{i N_{i}}$ are independent and identically distributed (i.i.d) random variables. Under the condition of Weibull envelope clutter, the outputs of square-law detector follow a
Weibull distribution with probability density functions (pdf) as

$$
f_{x}(x)=\frac{c_{i}}{2 b_{i}^{2}}\left(\frac{x}{b_{i}^{2}}\right)^{c_{i} / 2-1} \exp \left(-\left(\frac{x}{b_{i}^{2}}\right)^{c_{i} / 2}\right) .
$$

Here, $b_{i}^{2}$ is the scale parameter and $0.5 c_{i}$ is the shape parameter of clutter at the $i$ th sensor. In the Weibull clutter, (1), when $b_{i}^{2}$ and $0.5 c_{i}$ are unknown, in order that CFAR property holds, the detection is performed as, $[2,4]$,

$$
\left(\frac{x_{i 0}}{\widehat{b}_{i}^{2}}\right)^{\widehat{c}_{i} / 2} \underset{H_{0}}{\stackrel{H_{1}}{>}} g_{1}
$$

where $\widehat{b}_{i}^{2}$ and $\widehat{c}_{i}$ are estimation of the scale and shape parameters which can be formed by CFAR algorithm such as maximum likelihood (ML) [2] or order statistics (OS) [4]. The $g_{1}$ is also a complicated functions of desired $P_{\mathrm{FA}}$, the number of reference samples, and the method used for parameter estimation. In many situations the shape parameter changes slowly through the time. In this case the shape parameter can be estimated from the geometry and physical condition of the environment [13] or from a large number of reference samples [14]. In both cases the estimation error is enormous and it is almost realistic to assume $c_{i}$ as a known parameter [4]. By this assumption the detection strategy will be simplified as

$$
\frac{x_{i 0}}{\widehat{b}_{i}^{2}} \underset{H_{0}}{\stackrel{H_{1}}{>}} g_{1}^{2 / c_{i}}
$$

In this paper, we consider the situation in which the sensors locally perform CFAR processing before transmitting data to the fusion center. The data received by the fusion center, in this situation, is $s_{i}$, where

$$
s_{i}=\frac{x_{i 0}}{\widehat{b}_{i}^{2}}, \quad i=1,2 .
$$

At the fusion center, it was assumed that first, the received data from local sensor $s_{i}, i=1,2$, are weighted according to weighting functions and then are combined by deterministic rules to construct global test statistics. Thus, the global test statistics in the fusion center is made as follows:

$$
\begin{array}{ll}
G\left(w\left(s_{1}\right), w\left(s_{2}\right)\right) \geq T, & H_{1} \text { is true, } \\
G\left(w\left(s_{1}\right), w\left(s_{2}\right)\right)<T, & H_{0} \text { is true, }
\end{array}
$$

where operator $w(\cdot)$ is weighting functions in fusion center and operator $G(\cdot)$ is the global test statistics which is generated from $w\left(s_{i}\right), i=1,2$, by a predetermined fusion rule. Finally, the statistics $G$ is compared with threshold $T$ to decide about presence or absence of target, that is, $H_{1}$ or $H_{0}$ hypothesis, respectively. In this paper, we consider binary and fuzzy weighing functions, $w(\cdot)$, and use several fusion rules such an summation, algebraic product, algebraic sum, probabilistic OR, and Lukasiewicz t-conorm to produce global test statistics. 
2.1. Distributed System Based on Binary Weighting Functions and Summation Fusion Rule. In this subsection, we adapt the structure of distributed detectors such as Max, min and NTS in [9] based on global test statistics in (3) and summation fusion rule. These detectors, Max, min, and NTS, are defined as

Max:

$$
\max \left(s_{1}, s_{2}\right) \stackrel{H_{H_{0}}}{\stackrel{H_{1}}{<}} T_{M},
$$

$\min :$

$$
\min \left(s_{1}, s_{2}\right) \stackrel{H_{H_{0}}}{\stackrel{H_{1}}{>}} T_{m},
$$

NTS (sum):

$$
s_{1}+s_{2} \underset{H_{0}}{\stackrel{H_{1}}{>}} T_{s} .
$$

By considering the global test statistics in Max, min, and NTS as

$$
\begin{aligned}
G\left(w_{i}\left(s_{1}\right), w_{i}\left(s_{2}\right)\right) & =w_{i}\left(s_{1}\right)+w_{i}\left(s_{2}\right) \\
& =\alpha s_{1}+\beta s_{2} \stackrel{{ }_{H_{0}}}{\mathrm{H}_{1}} T,
\end{aligned}
$$

the weighting coefficient $\alpha$ and $\beta$ in max, min, and NTS can be obtained to be

$$
\begin{aligned}
& \operatorname{Max}: \begin{cases}\alpha=1, \beta=0, & \text { if } s_{1} \geq s_{2}, \\
\alpha=0, \beta=1, & \text { if } s_{1}<s_{2},\end{cases} \\
& \min : \begin{cases}\alpha=0, \beta=1, & \text { if } s_{1} \leq s_{2}, \\
\alpha=1, \beta=0, & \text { if } s_{1}>s_{2},\end{cases} \\
& \text { NTS : }\{\alpha=1, \beta=1\} .
\end{aligned}
$$

Therefore, in these distributed detectors, based on the new construction (5), it was found that, firstly, the received data from local sensors $s_{i}, i=1,2$, are weighted according to binary weighting functions, $\alpha$ and $\beta$, and then are combined by summation rule to construct the global test statistics. Generally, such binary weighting functions causes significant loss of information in fusion center. In order to reduce this imperfection, in the following subsection, we employ the soft weighing functions which can be implemented as a fuzzy membership functions. Then, the fuzzy weighted values are combined by fuzzy fusion rules, such as algebraic product, algebraic sum, probabilistic OR, and Lukasiewicz t-conorm, to produce global test statistics. In the following, based on local CFAR algorithm ML (OS), we named the distributed detectors based on (7), (8a)-(8c) as B-ML-S (B-OS-S), BML-m (B-OS-m), and B-ML-M (B-OS-M), respectively. The abbreviations denote, in order, type of weighting functions (binary (B) or fuzzy (F)), CFAR algorithm used in local detectors (ML or OS), and fusion rules to construct general test statistics (Max (M), min (m), NTS (S)).
2.2. Distributed System Based on Fuzzy Weighting Functions and Fuzzy Fusion Rule. As mentioned in the previous subsection, employing binary weighting functions in fusion center certainly causes significant loss of information in the fusion center. Recently, some authors have used fuzzy logic in detection to improve the system performance $[12,15-18]$. Indeed, in the fuzzy logic, binary functions are replaced with the soft functions. In this paper, we employ the soft weighing functions, $w\left(s_{i}\right)$, which can be implemented as a fuzzy membership functions, assigning membership to the hypothesis $H_{0}$. To evaluate weighting functions, we use uppercase letters to denote random variables and lowercase letters to denote their corresponding observation. The weighing functions, $w\left(s_{i}\right)$, is defined so that the weighted values are distributed uniformly on $[0,1]$ under $H_{0}$, as, [16],

$$
w\left(s_{i}\right)=\int_{s_{i}}^{\infty} f_{S_{i}}\left(z \mid H_{0}\right) d z=1-F_{S_{i}}\left(s_{i} \mid H_{0}\right),
$$

where, $f_{S_{i}}\left(s_{i} \mid H_{0}\right)$ is the pdf of random variable $S_{i}$ under hypothesis $H_{0}$ and can be expressed as, [19, page 187],

$$
\begin{aligned}
f_{S_{i}}( & \left.s_{i} \mid H_{0}\right) \\
\quad & =\int_{0}^{\infty} f_{X_{i 0}}\left(s_{i} \cdot y \mid H_{0}\right) \cdot f_{\widehat{B}_{i}^{2}}\left(y \mid H_{0}\right) \cdot|y| \cdot d y .
\end{aligned}
$$

In the following section, we obtain weighting functions based on ML or OS as local CFAR algorithm and use four fuzzy rules such as algebraic product, algebraic sum, probabilistic $\mathrm{OR}$, and Lukasiewicz t-conorm to construct the global test statistics.

2.2.1. Weighting Functions Based on ML CFAR Algorithm in Local Sensor. The ML CFAR algorithm uses maximum likelihood estimation of $b_{i}^{2}$ to reach CFAR property. When $c_{i}$ is assumed to be known and square law detector is employed, $\widehat{b}_{i}^{2}$ can be obtained as, [2],

$$
\widehat{b}_{i}^{2}=\left(\frac{1}{N_{i}} \sum_{j=1}^{N_{i}} x_{i j}^{c_{i} / 2}\right)^{2 / c_{i}}
$$

for the Weibull distribution, the pdf of $\widehat{B}_{i}^{2}$ can be obtained as, [20],

$$
\begin{aligned}
f_{\widehat{B}_{i}^{2}}(y)= & \frac{c_{i} N_{i}^{N_{i}}}{2\left(b_{i}^{c_{i}}\right)^{N_{i}} \Gamma\left(N_{i}\right)} y^{c_{i} / 2-1} \\
& \times\left(y^{c_{i} / 2}\right)^{N_{i}-1} \exp \left(-\frac{N_{i} y^{c_{i} / 2}}{b_{i}^{c_{i}}}\right),
\end{aligned}
$$


where, $\Gamma(\cdot)$ is the gamma functions. Substituting (1) and (12) into (10) results in

$$
\begin{aligned}
f_{s_{i}}\left(s_{i} \mid H_{0}\right)=\frac{c_{i}^{2} N_{i}^{N}}{4 b_{i}^{2} \Gamma\left(N_{\mathrm{i}}\right)} s_{i}^{c_{i} / 2-1} & \int_{0}^{\infty}\left(\frac{y}{b_{i}^{2}}\right)^{c_{i}\left(N_{i}+1\right) / 2-1} \\
& \times \exp \left(-\frac{N_{i} y^{c_{i} / 2}}{b_{i}^{c_{i}}}\right) \\
& \times \exp \left(-\left(\frac{s_{i} y}{b_{i}^{2}}\right)^{c_{i} / 2}\right) d y .
\end{aligned}
$$

By using the below transformation:

$$
v=\left(\frac{y}{b_{i}^{2}}\right)^{c_{i} / 2}
$$

we find

$$
f_{S_{i}}\left(s_{i} \mid H_{0}\right)=\frac{c_{i} N_{i}^{N_{i}}}{2 \Gamma\left(N_{i}\right)} s^{c_{i} / 2-1} \int_{0}^{\infty} v^{N_{i}} \exp (-\beta v) d v,
$$

where

$$
\beta=s_{i}^{c_{i} / 2}+N_{i}
$$

Using

$$
\int_{0}^{\infty} x^{n} \exp (-\delta x) d x=\frac{\Gamma(n+1)}{\delta^{n+1}},
$$

we obtain

$$
f_{S_{i}}\left(s_{i} \mid H_{0}\right)=\frac{c_{i}}{2} s_{i}^{c_{i} / 2-1}\left(1+\frac{\sqrt{s_{i}}{ }^{c_{i}}}{N_{i}}\right)^{-\left(N_{i}+1\right)} .
$$

Substituting (18) into (9) and integrating the resulting expression, we obtain

$$
w\left(s_{i}\right)=\left(1+\frac{{\sqrt{s_{i}}}^{c_{i}}}{N_{i}}\right)^{-N_{i}} .
$$

2.2.2. Weighting Functions Based on OS CFAR Algorithm in Local Sensor. In the OS algorithm, the observation samples, $x_{i j}$, are first rank ordered according to increasing magnitude. This rank ordered sequence is denoted by $x_{i(1)}, x_{i(2)}, \ldots, x_{i\left(N_{i}\right)}$ where $x_{i(r)}$ denote the $r$ th largest order statistics of $x_{i 1}, x_{i 2}, \ldots, x_{i N_{i}}$. From this sequence, $k_{i}$ th ordered value, $x_{i\left(k_{i}\right)}$, is selected as estimation of scale parameter. In the homogenous background case, the pdf $f_{x\left(k_{i}\right)}(\cdot)$ of random variable $x_{i\left(k_{i}\right)}$ is given by, [3],

$$
f_{x\left(k_{i}\right)}(y)=k_{i}\left(\begin{array}{c}
N_{i} \\
k_{i}
\end{array}\right)[F(y)]^{k_{i}-1} f(y)[1-F(y)]^{N_{i}-k_{i}},
$$

where $f(\cdot)$ and $F(\cdot)$ are the pdf and cumulative distribution functions (cdf) for the observations $x_{i j}$, respectively. The $f(\cdot)$ is given by (1), and $F(\cdot)$ is the corresponding cdf. Substituting $f(\cdot)$ and $F(\cdot)$ into (20) and using (14), we obtain

$$
\begin{aligned}
f_{S_{i}}\left(s_{i} \mid H_{0}\right)= & k_{i} \frac{c_{i}}{2}\left(\begin{array}{c}
N_{i} \\
k_{i}
\end{array}\right) s_{i}^{c_{i} / 2-1} \\
& \times \int_{0}^{\infty} v \cdot[1-\exp (-v)]^{k_{i}-1} \\
& \times \exp \left(-\left(N_{i}-k_{i}+1+s_{i}^{c_{i} / 2}\right) v\right) d v .
\end{aligned}
$$

Expanding $[1-\exp (-v)]^{k-1}$ by the binomial formula, the integration of (21) yields

$$
\begin{aligned}
f_{S_{i}}\left(s_{i} \mid H_{0}\right) \\
=k_{i} \frac{c_{i}}{2}\left(\begin{array}{c}
N_{i} \\
k_{i}
\end{array}\right) s_{i}^{c_{i} / 2-1} \\
\quad \times \sum_{l=0}^{k_{i}-1}(-1)^{l}\left(\begin{array}{c}
k_{i}-1 \\
l
\end{array}\right)\left(N_{i}-k_{i}+1+l+s_{i}^{c_{i} / 2}\right)^{-2} .
\end{aligned}
$$

Substituting (22) into (9) and integrating the resulting expression, we obtain

$$
w\left(s_{i}\right)=k_{i}\left(\begin{array}{c}
N_{i} \\
k_{i}
\end{array}\right) \sum_{l=0}^{k_{i}-1}(-1)^{l}\left(\begin{array}{c}
k_{i}-1 \\
l
\end{array}\right) \frac{1}{\left(N_{i}-k_{i}+1+l+s_{i}^{c_{i} / 2}\right)} .
$$

Using

$$
\sum_{l=0}^{k-1} \frac{(-1)^{l}}{(k-l-l) ! l !(y+l)}=\prod_{l=0}^{k-1} \frac{1}{(y+l)},
$$

consequently, the weighting functions $w\left(s_{i}\right)$ is obtained to be

$$
w\left(s_{i}\right)=\prod_{l=0}^{k_{\mathrm{i}}-1}\left(1+\frac{{\sqrt{s_{i}}}^{c_{i}}}{N_{\mathrm{i}}-l}\right)^{-1} .
$$

In the following, we use fusion rules such as algebraic product (AP), algebraic sum (AS), probabilistic OR (PO), and Lukasiewicz t-conorm (LTC) fusion to construct test global statistics. In the following the relationship between probability of false alarm $\left(P_{\mathrm{FA}}\right)$ and detection threshold, $T$, based on above fusion rules is derived.

Algebraic Product (AP). The algebraic product fuzzy fusion rule in fusion center is product of $w\left(s_{1}\right)$ and $w\left(s_{2}\right)$ as, [21],

$$
G=w\left(s_{1}\right) \times w\left(s_{2}\right) .
$$

The random variables of $w\left(s_{1}\right)$ and $w\left(s_{2}\right)$ are uniformly distributed in $[0,1]$, [19, page 139]. Thus, we have, [19, page 205],

$$
f_{G}(y)=-\ln (y), \quad 0 \leq y \leq 1
$$


Algebraic Sum (AS). The algebraic sum fuzzy fusion rule in fusion center is defined by, [22],

$$
G=w\left(s_{1}\right)+w\left(s_{2}\right) .
$$

The pdf $f_{G}(y)$ can be obtained as, [19, page 184],

$$
f_{G}(y)= \begin{cases}y, & 0 \leq y<1 \\ 2-y, & 1 \leq y \leq 2 .\end{cases}
$$

Probabilistic OR (PO). The probabilistic OR (sum) fuzzy fusion rule in fusion center is defined by the relation, [21],

$$
G=w\left(s_{1}\right)+w\left(s_{2}\right)-w\left(s_{1}\right) \times w\left(s_{2}\right) .
$$

Similar to (26), the pdf $f_{G}(y)$ can be obtained as

$$
f_{G}(y)=-\ln (1-y), \quad 0 \leq y \leq 1 .
$$

Lukasiewicz t-conorm (LTC). The Lukasiewicz t-conorm fuzzy fusion rule in fusion center is defined by the relation, [22],

$$
G=\min \left(w\left(s_{1}\right)+w\left(s_{2}\right), 1\right) .
$$

The cdf of (32) can be obtained as

$$
F_{G}(y)= \begin{cases}0.5 y^{2}+0.5, & y \geq 1, \\ 0.5 y^{2}, & y<1 .\end{cases}
$$

From (9), it is clear that $w\left(s_{i}\right)$ is a decreasing functions as cdf $F_{S_{i}}\left(s_{i} \mid H_{0}\right)$ is an increasing functions. On other hand, since random variables of $w\left(s_{1}\right)$ and $w\left(s_{2}\right)$ are also uniformly distributed in $[0,1]$, it is easy to prove that $G$ in (26), (28), (30), and (32) is also a decreasing functions of $s_{i}$. Thus, the global test statistics in fusion center can be written as

$$
\begin{aligned}
& G \leq T, \quad H_{1} \text { is true, } \\
& G>T, \quad H_{0} \text { is true. }
\end{aligned}
$$

Therefore, the $P_{\mathrm{FA}}$ in the fusion centre can be represented as

$$
P_{\mathrm{FA}}=P\left\{G \leq T \mid H_{0}\right\}=F_{G}(T) .
$$

After solving (35) for algebraic product [12], algebraic sum, probabilistic OR [12], and Lukasiewicz t-conorm fuzzy rules, the relationship between $P_{\mathrm{FA}}$ and $T$ can be obtained, respectively, as

$$
\begin{gathered}
P_{\mathrm{FA}, \mathrm{AP}}=T(1-\ln (T)), \\
P_{\mathrm{FA}, \mathrm{AS}}=\int_{0}^{T} f_{G}(y) d y=\int_{0}^{T} y d y=0.5 T^{2}, \\
P_{\mathrm{FA}, \mathrm{PO}}=T+(1-T) \cdot \ln (1-T), \\
P_{\mathrm{FA}, \mathrm{LTC}}=F_{G}(T)=0.5 T^{2} .
\end{gathered}
$$

In the following, based on local CFAR algorithm ML (OS) and fuzzy (F) weighting functions in fusion center, the resulting detectors are named as F-ML-AP (F-OS-AP), F-ML-AS (FOS-AS), F-ML-PO (F-OS-PO), and F-ML-LTC (F-OS-LTC).

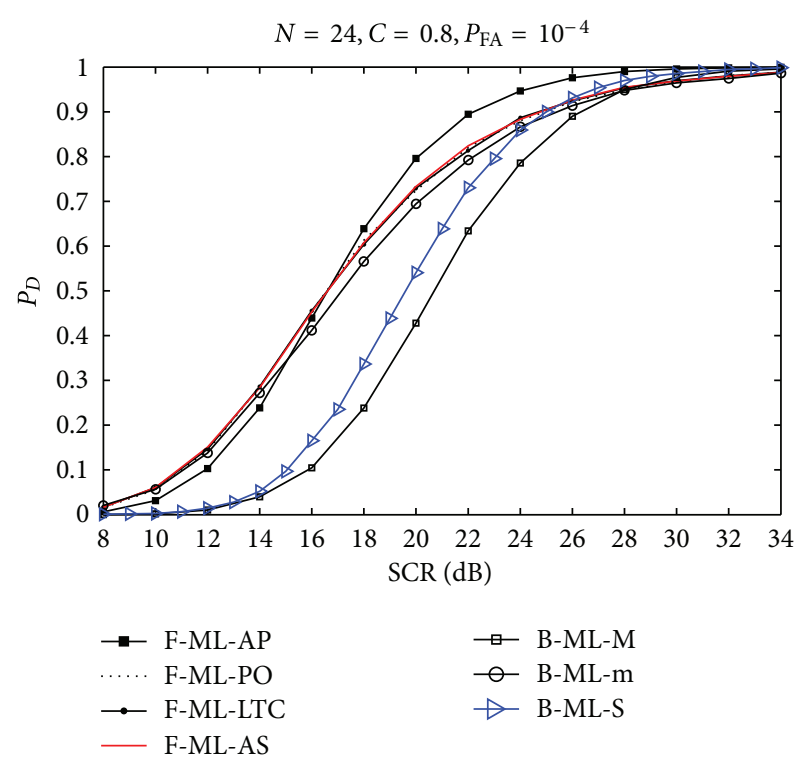

FIGURE 1: PD versus SCR performance of distributed detector based on ML CFAR algorithm in local sensors when $C=0.8$.

\section{Performance Study}

While the clutter distribution is Weibull, it is not easy to find closed form expressions of the $P_{D}$ for many detectors. Even when finding them, usually they are too complicated to produce any insight about the behavior of the detector. Therefore, the computer simulation is a usual method for evaluating the performance of detectors. In the simulations of this section, as many similar works, we consider the Rayleigh model for fluctuating targets, representing Swerling I (SWI) and Swerling II (SWII) (single pulse assumption) and we examine and compare the $P_{D}$ and $P_{\mathrm{FA}}$ performance of the mentioned distributed detector with each other. Unless otherwise stated, we consider the symmetrical case where all the parameters of the local detectors are assumed to be the same, that is, $N_{1}=N_{2}=N=24, C=c_{1}=c_{2}, \mathrm{SCR}_{1}=$ $\mathrm{SCR}_{2}=\mathrm{SCR}$, and $k_{1}=k_{2}=k=20$ (OS CFAR local detectors case).

3.1. Homogenous Clutter Situations. The detection performance of all detectors based on OS and ML CFAR algorithms, in the case of homogenous clutter, are shown in Figures 16. In Figure 1, the probabilities of detection $\left(P_{D}\right)$ against the signal-to-clutter ratio (SCR) of the F-ML-AP, F-MLPO, F-ML-LTC, F-ML-AS, B-ML-M, B-ML-m, and B-ML-S distributed detectors are shown. The superior performance of the F-ML-AP detector over the B-ML-S, B-ML-M, F-MLPO, F-ML-LTC, F-ML-AS, and B-ML-m can be observed. Figure 2 shows the detection performance of the distributed system where the CFAR algorithm in local detectors is OS. The same observation as in Figure 1 can be made. Figures 3 and 4 give the probability of detection in distributed detectors based on ML and OS CFAR algorithms in local detectors, corresponding to the shape parameters $C=4$ and $C=2$, respectively. From Figures $1-4$, it is observed 


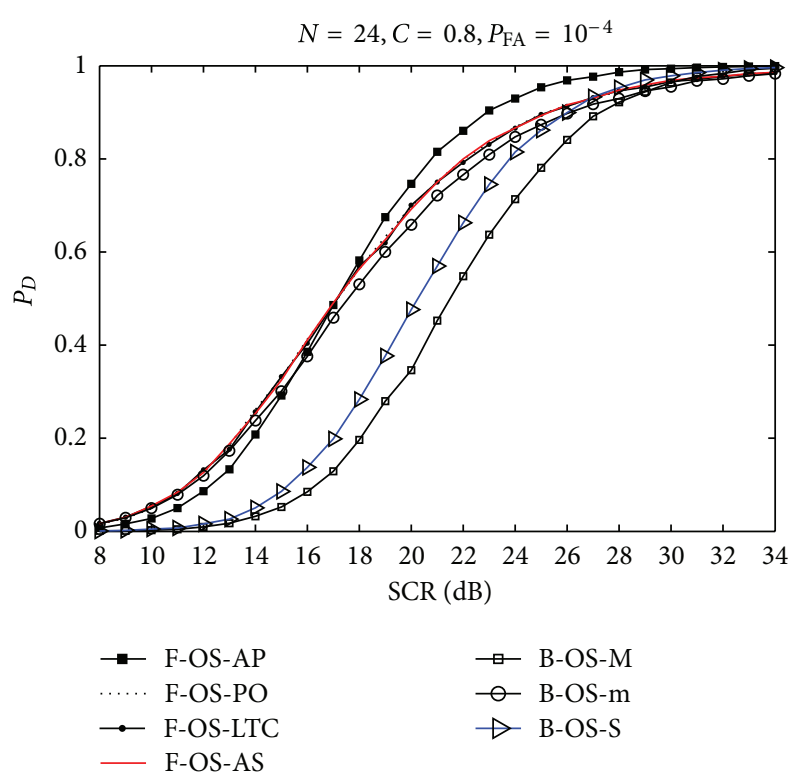

FIGURE 2: PD versus SCR performance of distributed detector based on OS CFAR algorithm in local sensors when $C=0.8$.

that the F-ML-AP (F-OS-AP) performs better than other mentioned distributed detectors based on ML (OS) CFAR algorithms in local detectors. For large SCR and for large shape parameter values (Figure 3), the B-ML-M and B-ML$\mathrm{S}$ perform close to the F-ML-AP whereas for low value of the shape parameters (Figures 1, 2, and 4), the B-ML-S (BOS-S) does better than B-ML-M (B-OS-M). To compare the performance of distributed detectors based on OS and ML CFAR algorithms, let us consider Figure 5. In this Figure, we present the performance comparison of the F-ML-AP and FOS-AP for three values of the shape parameter, $C=2,0.8$, and 0.5 . As expected, the better performance of the F-MLAP over the F-OS-AP can be observed. From this Figure, we also observe that the probabilities of detection become better when $C$ is higher, which is expected since in this case the clutter distribution approaches a Rayleigh pdf. The effect of unequal received SCR in two sensors on the detection performance was evaluated in Figure 6. In Figure 6, the SCR at sensor 1 is $\mathrm{SCR}_{1}=20 \mathrm{~dB}$, and the $\mathrm{SCR}$ in sensor $2\left(\mathrm{SCR}_{2}\right)$ ranges from $-20 \mathrm{~dB}$ to $40 \mathrm{~dB}$. For $\mathrm{SCR}_{2}$ from $-20 \mathrm{~dB}$ through 0 , the better performance of the B-ML-M detector followed by the B-ML-S, over the F-ML-AP, F-ML-PO, F-ML-LTC, F-ML-AS, and B-ML-m, can be observed. But, for $\mathrm{SCR}_{2}>$ $0 \mathrm{~dB}$, this figure demonstrates superiority of F-ML-AP over all others. From simulation in this section, we observe that the F-ML-PO, F-ML-LTC, and F-ML-AS detectors perform exactly as each other.

3.2. Multiple Target Situation. In the multiple target situations, the local CFAR algorithms overestimate the scale parameter $\left(b_{i}^{2}\right)$ of clutter. As a result, the probability of false alarm $\left(P_{\mathrm{FA}}\right)$ decreases to a lower value than the design value. This is acceptable since it does not exceed the design $P_{\mathrm{FA}}$ value. But in addition to the $P_{\mathrm{FA}}$, the $P_{D}$ also decreases

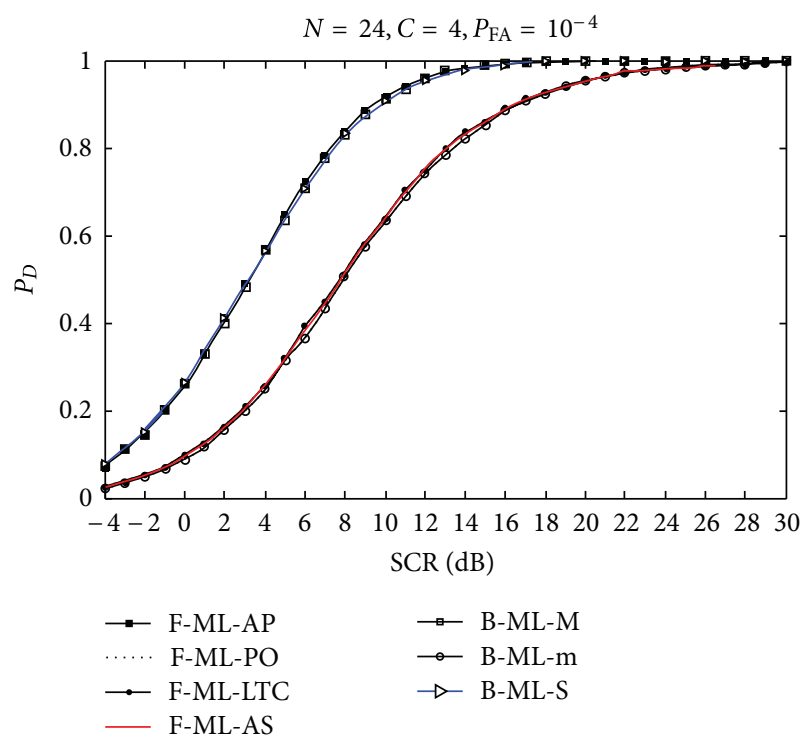

FIGURE 3: PD versus SCR performance of distributed detector based on ML CFAR algorithm in local sensors when $C=4$.

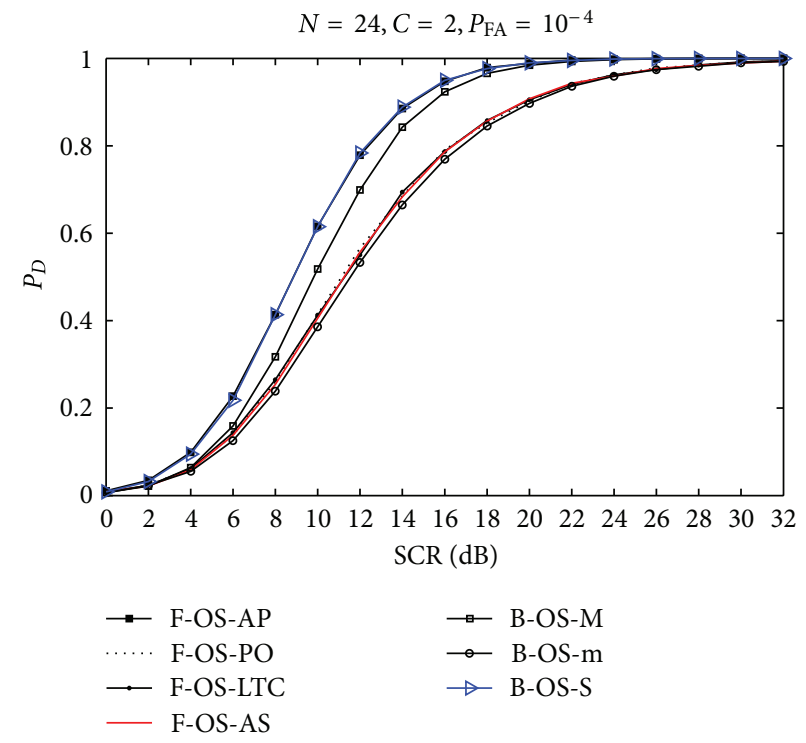

FIGURE 4: PD versus SCR performance of distributed detector based on OS CFAR algorithm in local sensors when $C=2$.

resulting in the so-called masking effect. So the main concern in the multiple target situations is to reduce the masking effect due to the interfering target. In this case, we analyzed the detection performance of the mentioned detectors in the multiple target situations. The test cell target and interfering targets were assumed to have same model and average power, that is, SCR = ICR (interference to clutter ratio (ICR)). Figures 7 and 8 show $P_{D}$ as a functions of SCR when the CFAR algorithms in local detectors are ML and OS, respectively. In Figures 7 and 8 , it was assumed that sensor 1 observed no interfering target $\left(b_{1}=0\right)$ but sensor 2 observed 4 interfering targets $\left(b_{2}=4\right)$ within their reference windows. From these 


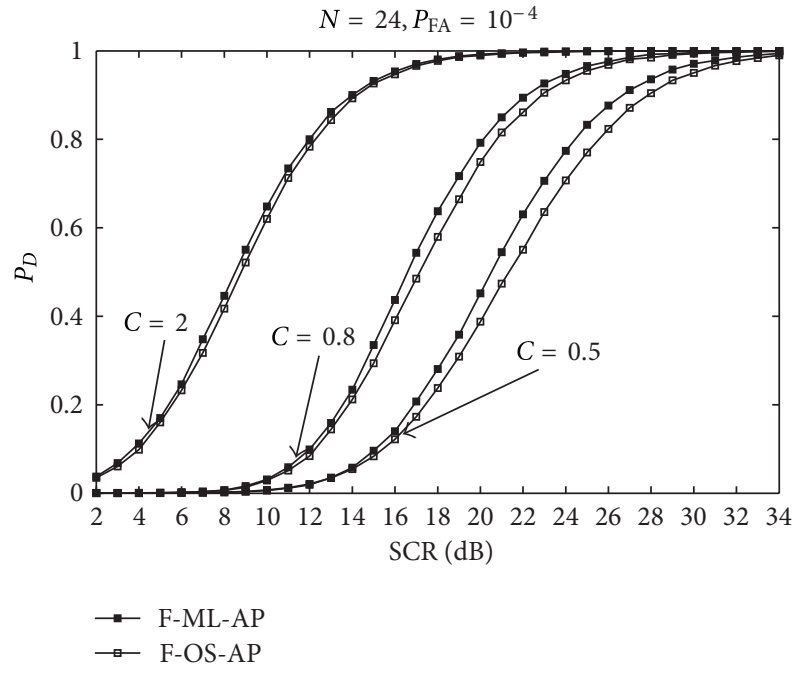

Figure 5: Performance comparison between distributed detector based on ML and OS CFAR algorithms in local sensors for different values of the shape parameters.

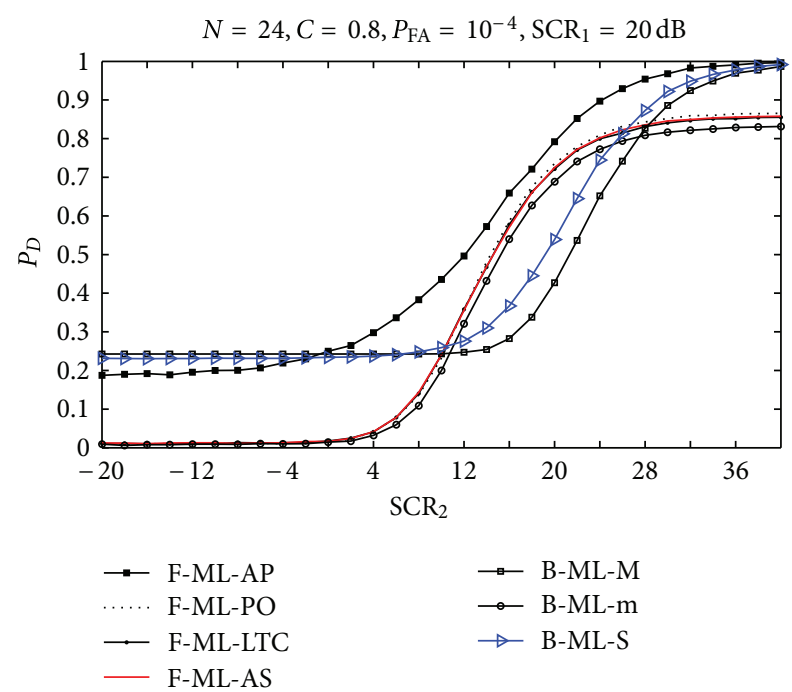

FIGURE 6: PD versus $\mathrm{SCR}_{2}$ performance of distributed detector based on ML CFAR algorithm in local sensors when $\mathrm{SCR}_{1}=20 \mathrm{~dB}$.

figures, the better performance of the F-ML-AP (F-OS-AP) followed by the B-ML-S (B-OS-S) and B-ML-M (B-OS-M), particularly for $P_{D}$ greater than 0.9 , over the F-ML-PO (FOS-PO), F-ML-LTC (F-OS-LTC), F-ML-AS (F-OS-AS), and B-ML-m (B-OS-m), can be observed. From these figures, we also observe that in multiple target situations the distributed detector based on OS CFAR algorithm perform better than the distributed detector based on ML CFAR algorithm in local detectors.

In Figures 9 and 10, we studied the effect of an increase in the number of interfering targets on the detection performance of distributed detectors based on OS and ML CFAR algorithms in local detectors, respectively. In these figures the number of interfering targets at sensor 1 is $b_{1}=2$, and

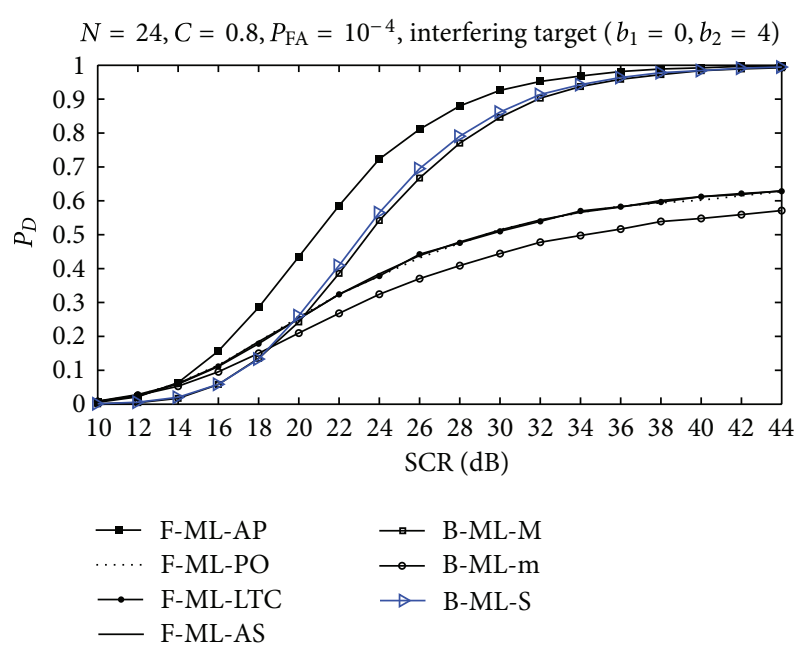

FIGURE 7: PD versus SCR performance of distributed detector based on ML CFAR algorithm in local sensors when interfering targets are presented.

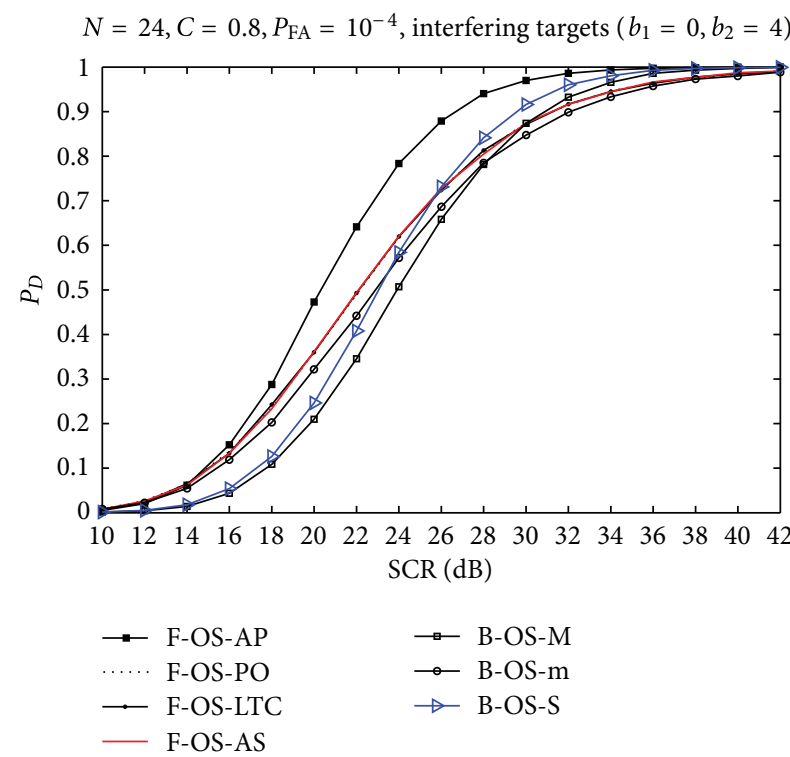

FIGURE 8: PD versus SCR performance of distributed detector based on OS CFAR algorithm in local sensors when interfering targets are presented.

the number of interfering targets in sensor $2\left(b_{2}\right)$ ranges from 0 to 10. From Figure 9, we observe a superior detection performance of F-ML-AP over all the other detectors in variation range of $b_{2}$. In Figure 10, for $b_{2}$ from 0 through 4 , the F-OS-AP detector performs better than F-OS-PO, FOS-LTC, F-OS-AS, B-OS-m, B-OS-S, and B-OS-M CFAR detectors. But, for $b_{2}$ greater than 4 , a significant degradation in detection performance occurs for these detectors. This is expected, because the maximum number of tolerable interfering targets in OS based algorithm depends on the selected rank and in this case equals to $4(N-k=4)$. In Figure 10 , it can also be seen that for $b_{2}>8$, the F-OS-AP has lower 


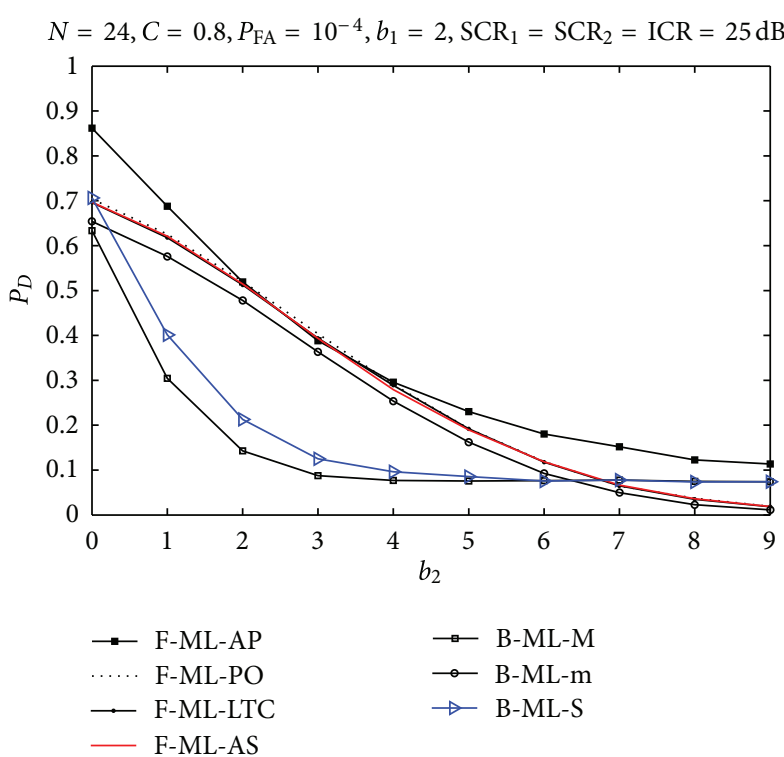

FIGURE 9: Detection performance of distributed detector based on ML CFAR algorithm versus number $b_{2}$ of interfering target at sensor 2 when $b_{1}=2$.

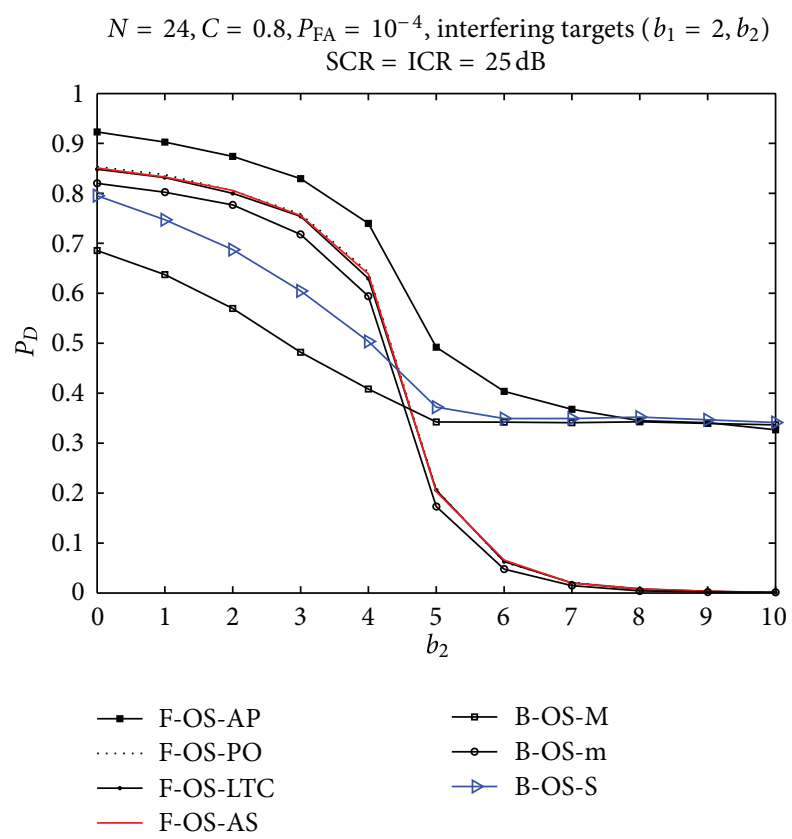

FIGURE 10: Detection performance of distributed detector based on OS CFAR algorithm versus number $b_{2}$ of interfering target at sensor 2 when $b_{1}=2$.

$P_{D}$ as compared with B-OS-M and B-OS-S but has a much better performance than the other detectors.

3.3. Clutter Edge Environment. In the radar detection, in order to prevent saturation of plan position indicator (PPI) and degradation of detector performance, the false alarm rate must be maintained less than or equal to a designed value. But the nonhomogenous situations practically exist and therefore

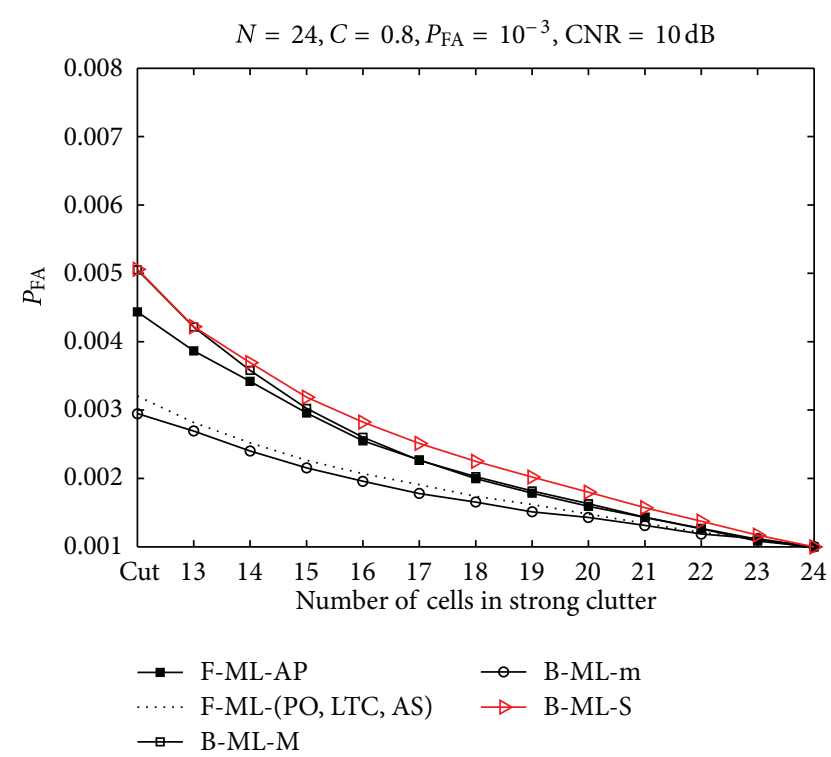

FIgURe 11: $P_{\text {FA }}$ performance of detectors based on ML CFAR processor versus number of reference windows cells in sensor 1 filled with strong clutter when test cell (cut) and reference window cells in sensor 2 are filled with strong clutter, $N=24, \mathrm{CNR}=10 \mathrm{~dB}$.

the $P_{\mathrm{FA}}$ exceeds the design value. In this subsection we evaluate one of these non-homogenous situations named clutter edge. In practice, a clutter edge situation is an abrupt clutter transition which may represent the boundary of a rain storm, flock, or a precipitation area. In the following, the clutter samples preceding the clutter edge are supposed to have strong clutter with power equal to $P_{1}$, and the samples following the clutter edge are supposed to have power equal to $P_{2}\left(P_{1}>P_{2}\right)$.

Figures 11 and 12 show the $P_{\mathrm{FA}}$ of the above detectors in the clutter edge environment when the ratio between the clutter power is given by the clutter to noise ratio $(\mathrm{CNR})$ parameter that is equal to $10 \mathrm{~dB}$ (i.e., $\mathrm{CNR}=P_{1} / P_{2}=$ $10 \mathrm{~dB}$ ). In clutter edge environment mentioned here the strong clutter filled half of the cells of reference windows in sensor 1 and the test cell is also in strong clutter (which is the worst case corresponding to maximum increase $\left.P_{\mathrm{FA}}\right)$, then the clutter continued to fill the remaining reference windows cells in sensor 1; in these situations all the reference cells and test cells in sensor 2 were filled with clutter with power $P_{1}$, homogenous situation. From the performance curves showed in Figures 11 and 12, the better $P_{\mathrm{FA}}$ control of B-ML-m (B-OS-m) followed by the F-ML-PO (F-OS-PO), F-ML-LTC (F-OS-LTC), F-ML-AS (F-OS-AS), over the F-ML-AP (FOS-AP), B-ML-M (B-OS-M), and B-ML-S (B-OS-S), can be observed.

\section{Summary and Conclusions}

In this paper, we have developed two types of distributed CFAR detector using distributed sensors in Weibull clutter. In these detectors, it has been assumed that the scale parameters of clutters at the local sensors are unknown and 


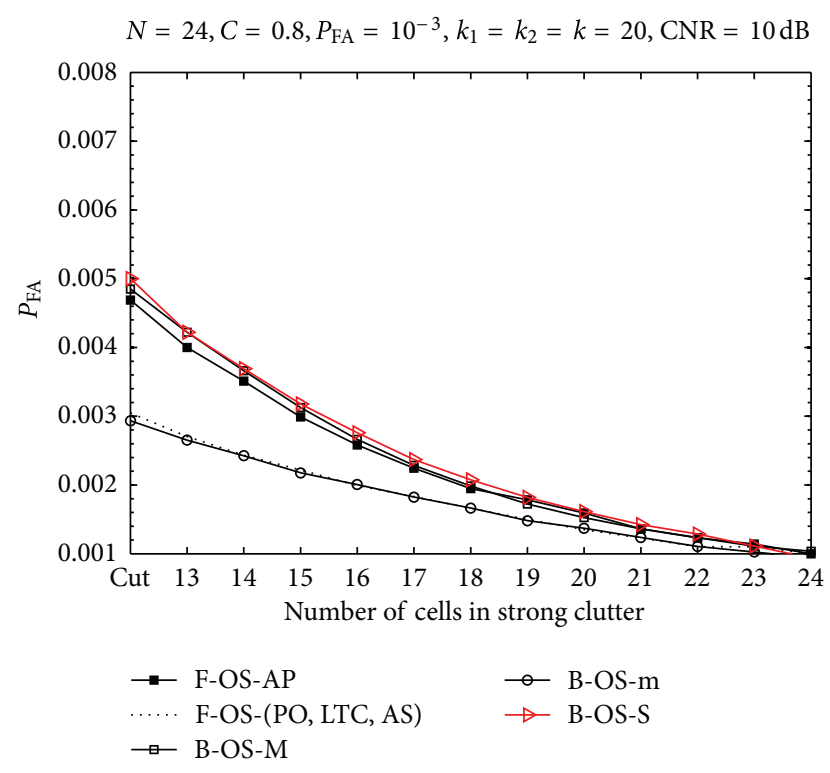

FIGURE 12: $P_{\mathrm{FA}}$ performance of detectors based on OS CFAR processor versus number of reference windows cells in sensor 1 filled with strong clutter when test cell (cut) and reference window cells in sensor 2 are filled with strong clutter, $N=24, k_{1}=k_{2}=k=20$, $\mathrm{CNR}=10 \mathrm{~dB}$.

each local detector performed ML (OS) CFAR processing before transmitting data to the fusion center. In the binary type, we have examined the distributed detectors with binary weighting functions in fusion center such as B-ML-M (BOS-M), B-ML-m (B-OS-m), and B-ML-S (B-OS-S) CFAR. In the fuzzy types the distributed detectors with fuzzy weighting functions such as F-ML-AP (F-OS-AP), F-ML-PO (F-OS-PO), F-ML-LTC (F-OS-LTC), and F-ML-AS (F-OSAS) have been proposed. Moreover, for the Weibull clutter, the expression of the weighting functions, based on ML and OS CFAR processor in local detectors, is obtained.

We conclude from the study of a two-sensor system that for the homogenous situation Weibull clutter, the detection performance of the F-ML-AP is considerably better than all other distributed detectors. When the two sensors do not see the same $\mathrm{SCR}$ (i.e., $\mathrm{SCR}_{1} \neq \mathrm{SCR}_{2}$ ), the simulation results have also indicated robust and superior performance of F-ML-AP (F-OS-AP).

Next, the problem of CFAR detection in multiple targets and a nonhomogenous clutter background has been addressed. In multiple targets situation, the results have demonstrated better performance of distributed detectors based on OS CFAR algorithm than the distributed detectors based on ML CFAR algorithm. In this case, the F-OS-AP and F-ML-AP detectors result in robust performance in presence of several interfering targets more than the other distributed detectors based on OS and ML CFAR algorithm, respectively. In the presence clutter edge in reference cells, the B-MLm (B-OS-m), F-ML-PO (F-OS-PO), F-ML-LTC (F-OS-LTC), and F-ML-AS (F-OS-AS) have controlled probability of false alarm better than all other detectors. In this case, the FML-AP (F-OS-AP) has better $P_{\mathrm{FA}}$ than the B-ML-M (B-OS$\mathrm{M})$ and B-ML-S (B-OS-S). Simulation results also showed that the algebraic sum, probabilistic OR, and Lukasiewicz $\mathrm{t}$-conorm rules perform exactly as each other, so we can select F-ML-AS (F-OS-AS) as the best detector within these detectors because this detector needs lower number of operations than F-ML-PO (F-OS-PO) and F-ML-LTC (F-OS-LTC) detectors.

Through the paper we assumed independent samples. This assumption is not valid in some situations (especially in sea clutter at high sea states), therefore a main topic for the further research will be investigating the effect of correlated samples on the proposed detectors.

\section{References}

[1] H. M. Finn and R. S. Johnson, "Adaptive detection mode with threshold control as a function of spatially sampled clutter-level estimate," RCA Review, vol. 29, no. 3, pp. 414-464, 1968.

[2] R. Ravid and N. Levanon, "Maximum-likelihood CFAR for Weibull background," IEE Proceedings F, vol. 139, no. 3, pp. 256-264, 1992.

[3] H. Rohling, "Radar CFAR thresholding in clutter and multiple target situations," IEEE Transactions on Aerospace and Electronic Systems, vol. 19, no. 4, pp. 608-621, 1983.

[4] N. Levanon and M. Shor, "Order statistics CFAR for Weibull background," IEE Proceedings F, vol. 137, no. 3, pp. 157-162, 1990.

[5] A. R. Elias-Fuste, M. G. G. de Mercado, and E. de los Reyes Davo, "Analysis of some modified ordered statistic CFAR: OSGO and OSSO CFAR," IEEE Transactions on Aerospace and Electronic Systems, vol. 26, no. 1, pp. 197-202, 1990.

[6] M. Barkat and P. K. Varshney, "Decentralized CFAR signal detection," IEEE Transactions on Aerospace and Electronic Systems, vol. 25, no. 2, pp. 141-149, 1992.

[7] M. K. Üner and P. K. Varshney, "Distributed cfar detection in homogeneous and nonhomogeneous backgrounds," IEEE Transactions on Aerospace and Electronic Systems, vol. 32, no. 1, pp. 84-97, 1996.

[8] H. Amirmehrabi and R. Viswanathan, "A new distributed constant false alarm rate detector," IEEE Transactions on Aerospace and Electronic Systems, vol. 33, no. 1, pp. 85-97, 1997.

[9] J. Guan, Y. N. Peng, Y. He, and X. W. Meng, "Three types of distributed CFAR detection based on local test statistic," IEEE Transactions on Aerospace and Electronic Systems, vol. 38, no. 1, pp. 278-288, 2002.

[10] C. H. Gowda and R. Viswanathan, "Performance of distributed cfar test under various clutter amplitudes," IEEE Transactions on Aerospace and Electronic Systems, vol. 35, no. 4, pp. 1410-1419, 1999.

[11] F. Gini, F. Lombardini, and L. Verrazzani, "Robust nonparametric multiradar CFAR detection against non-Gaussian spiky clutter," IEE Proceedings F, vol. 144, no. 4, pp. 131-140, 1997.

[12] Z. Hammoudi and F. Soltani, "Distributed CA-CFAR and OSCFAR detection using fuzzy spaces and fuzzy fusion rules," IEE Proceedings: Radar, Sonar and Navigation, vol. 151, no. 3, pp. 135-142, 2004.

[13] F. E. Nathanson, J. P. Reilly, and M. N. Cohen, Radar Design Principles, SciTech Publishing, New York, NY, USA, 2nd edition, 1999. 
[14] G. de Miguel and J. R. Casar, "CFAR detection for Weibull and other log-log-linear tail clutter distributions," IEE Proceedings: Radar, Sonar and Navigation, vol. 144, no. 2, pp. 64-70, 1997.

[15] S. W. Leung and J. W. Minett, "CFAR data fusion using fuzzy integration," in Proceedings of the 5th IEEE International Conference on Fuzzy Systems, pp. 1291-1295, New Orleans, La, USA, September 1996.

[16] S. W. Leung and J. W. Minett, "The use of fuzzy spaces in signal detection," Fuzzy Sets and Systems, vol. 114, no. 2, pp. 175-184, 2000.

[17] S. W. Leung and J. W. Minett, "Signal detection using fuzzy membership function," in Proceedings of the International AMSE Conference SYS'95, pp. 89-92, Brno, Czech Republic, July 1995.

[18] A. Zaimbashi, M. R. Taban, M. M. Nayebi, and Y. Norouzi, "Weighted order statistic and fuzzy rules CFAR detector for Weibull clutter," Signal Processing, vol. 88, no. 3, pp. 558-570, 2008.

[19] A. Papoulis, Probability Random Variables and Stochastic Processes, McGraw-Hill, New York, NY, USA, 4th edition, 2001.

[20] V. Anastassopoulos and G. A. Lampropoulos, "Optimal CFAR detection in Weibull clutter," IEEE Transactions on Aerospace and Electronic Systems, vol. 31, no. 1, pp. 52-64, 1995.

[21] B. Butkiewicz, "Comparison of operations used for fuzzy modeling," in Proceedings of the IEEE International Conference on Fuzzy Systems, pp. 681-684, Budapest, Hungery, July 2004.

[22] D. Dubois, E. Hullermeier, and A. Prade, "A note on quality measures for fuzzy association rules," in Proceedings of the 10th International Fuzzy System Association Word Congress, vol. 2715 of Lecture Notes in Artificial Intelligence, pp. 648-677, Springer, Istambul, Turkey, 2003. 

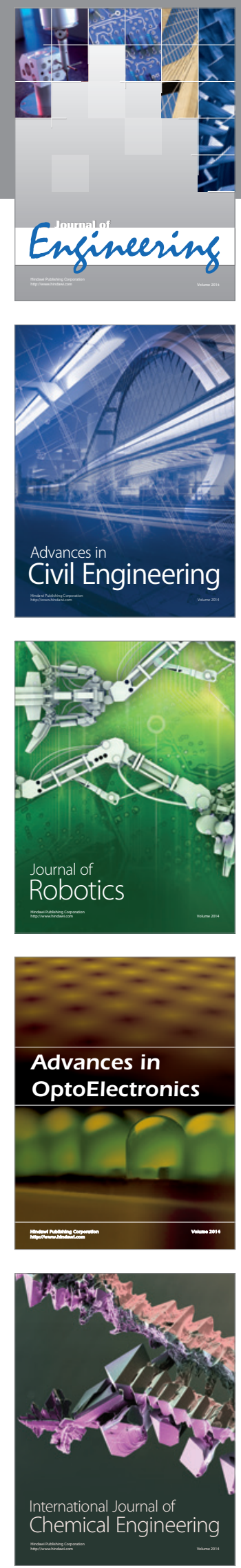

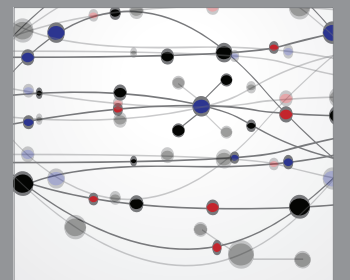

The Scientific World Journal
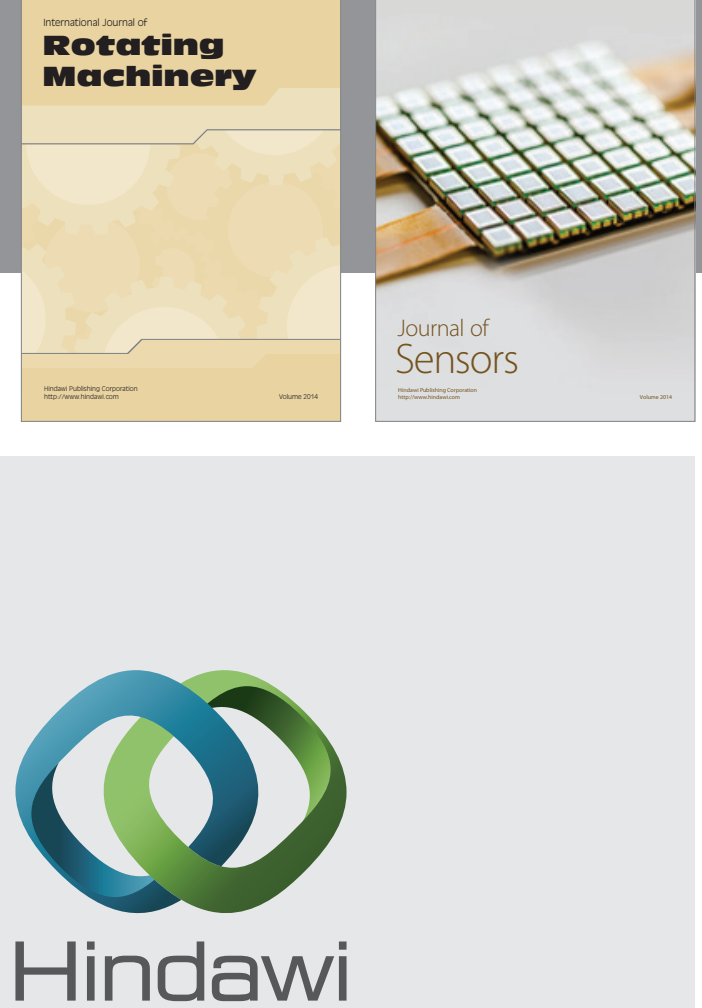

Submit your manuscripts at http://www.hindawi.com
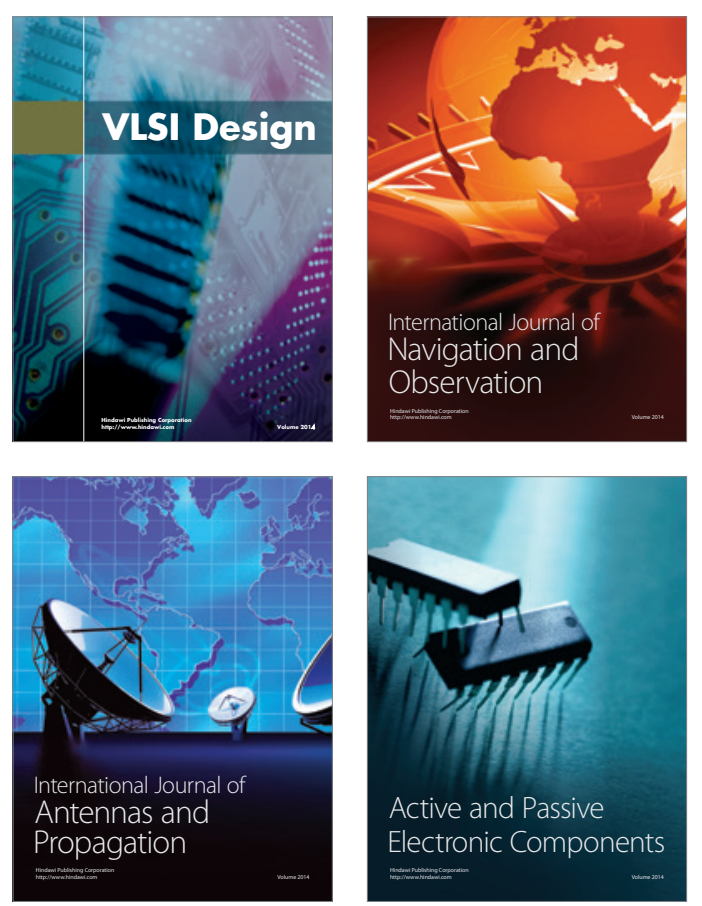
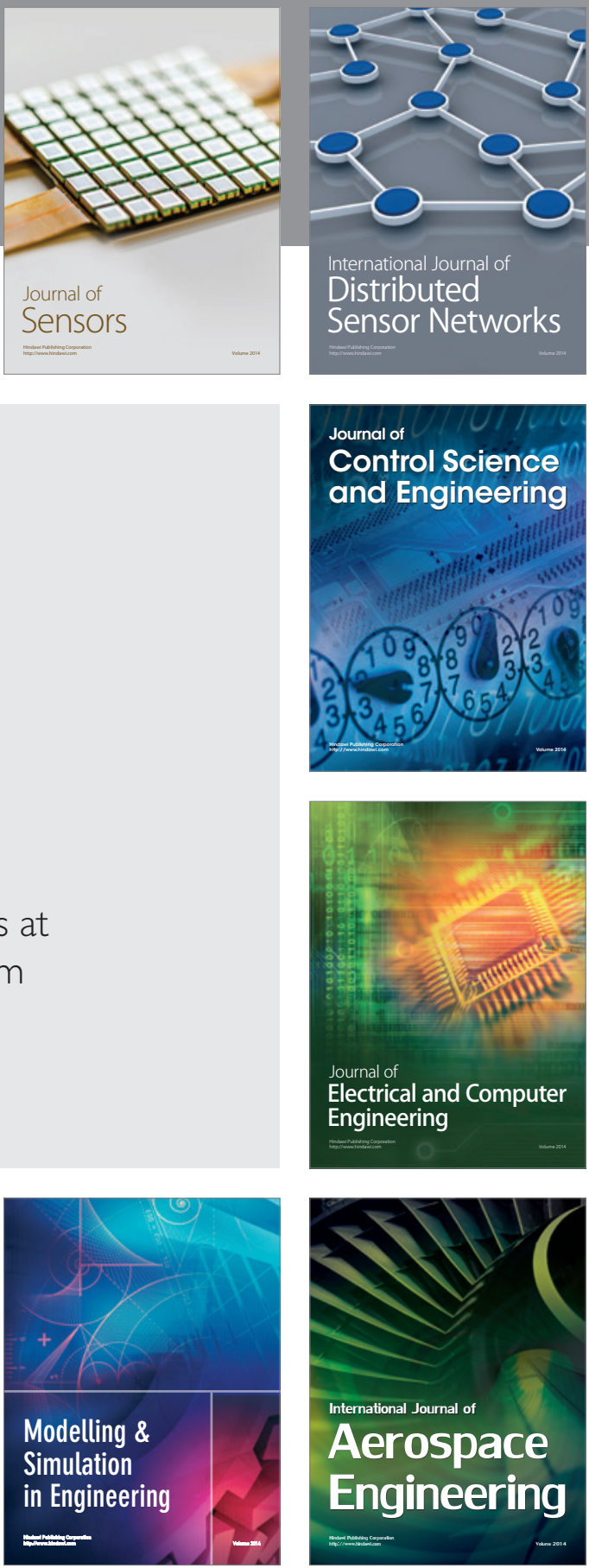

Journal of

Control Science

and Engineering
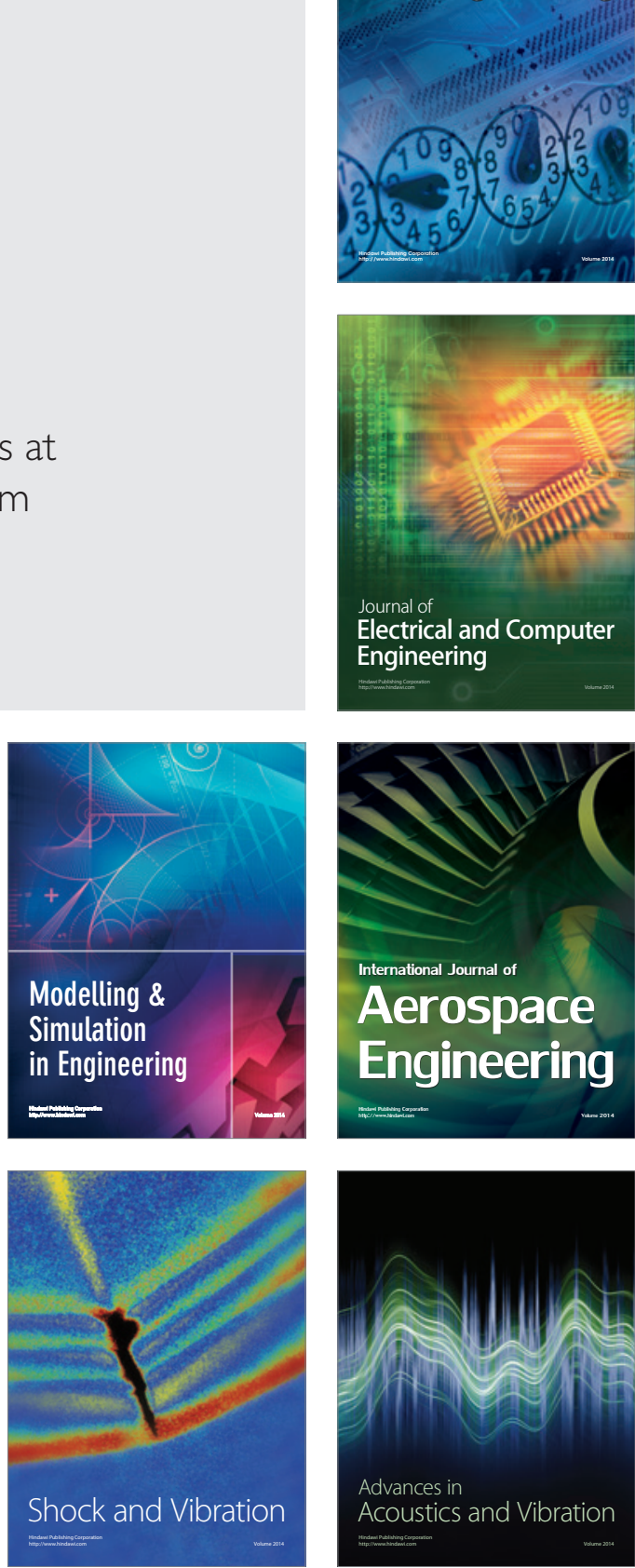